\title{
Endothelial cells of extremely premature infants display impaired immune response after proinflammatory stimulation
}

\author{
Lukas Wisgrill ${ }^{1}$, Martina Muck ${ }^{1}$, Isabelle Wessely ${ }^{1}$, Angelika Berger ${ }^{1}$, Andreas Spittler ${ }^{2}$, Elisabeth Förster-Waldl ${ }^{1}$ and \\ Kambis Sadeghi ${ }^{1}$
}

BACKGROUND: Endothelial cells (ECs) exert immunological functions such as production of proinflammatory cytokines/ chemokines as well as facilitation of extravasation of immune cells into infected tissue. Limited data are available on the functionality of ECs from extremely preterm neonates during infection. Accordingly, the aim of our study was to investigate the immune response of premature ECs after proinflammatory stimulation.

METHODS: Cell adhesion receptors' expression and function, nuclear factor 'kappa-light-chain-enhancer' of activated B-cells (NFkB) signaling, and chemokine production were analyzed in umbilical cord ECs from extremely preterm and term neonates after proinflammatory stimulation.

RESULTS: P-selectin and E-selectin surface expression as well as NFKB signaling were lower after lipopolysaccharide (LPS) stimulation in premature ECs. Preterm ECs exhibited lower, but significant, cell-adhesive functions after LPS stimulation compared with term ECs. CCL2/CXCL8 chemokine secretion was significantly upregulated after proinflammatory stimulation in both groups. CXCL10 production was significantly increased in term but not in preterm ECs upon stimulation with tumor necrosis factor compared with unstimulated ECs. CONCLUSION: Extremely premature ECS showed partly reduced expression levels and function of cell adhesion molecules. Both NFkB signaling and chemokine/cytokine production were reduced in premature ECs. The diminished endothelial proinflammatory immune response might result in impaired infection control of preterm newborns rendering them prone to severe infection.

B acterial infections and associated inflammation are a leading cause of neonatal mortality and morbidity, especially in extremely premature infants born before 28 weeks of gestational age (GA). Those high-risk neonates display a significantly increased risk of acquiring severe bacterial infections associated with long-term sequelae. The prevalence of neonatal sepsis is inversely correlated with GA and birth weight, indicating functional immaturities of the fetal and neonatal immune system (1-3). Endothelial cells (ECs) have an important role in the immune response against various pathogens. ECs express membrane-bound receptors for growth factors, coagulant and anticoagulant proteins, lipoproteins, metabolites, and hormones as well as receptors that govern cell-cell and cell-matrix interactions. More importantly, ECs seem to act as important mediators in the immune response by expressing both Toll-like receptors (TLRs) and Nod-like receptors, as well as chemokine receptors (4-7). In case of infection, ECs are important inducers of chemotaxis as they are capable of secreting proinflammatory cytokines as well as chemokines, thus delegating further activation, migration, and extravasation of leukocytes to the site of infection (8).

To date, only limited data are available on the functionality of ECs and their role in the immune response of extremely preterm neonates born before 28 weeks of GA. Therefore, we aimed to gain deeper insights into the immune response of human umbilical vein endothelial cells (HUVECs) isolated from premature neonates born before 28 weeks of GA after proinflammatory stimulation.

\section{METHODS}

\section{Study Population and Umbilical Cord Sampling}

Extremely premature infants $(n=7$, mean GA $24.57 \pm 1.14$ weeks, mean birth weight $670 \pm 98 \mathrm{~g}$ ) were compared with term newborns $(n=7$, mean GA $39.14 \pm 2.86$ weeks, mean birth weight $3,002 \pm 290 \mathrm{~g}$ ). The umbilical cord was collected under sterile conditions into phosphate-buffered saline (PBS) tubes after cesarean section and processed immediately after collection. Preterm infants with clinical signs of infection, elevated infection parameters (Creactive protein, interleukin (IL)-6, or white blood cell count), or chorioamnionitis were excluded. The reason for premature birth was mainly cervical insufficiency $(n=4)$, pathological doppler signal $(n=2)$, and non-inhabitable contractions without clinical and laboratory signs of infection of the neonate $(n=1)$. One premature twin birth was included in the study. All mothers received antenatal corticosteroids for lung maturation and magnesium for neuroprotection. The study was approved by the local ethics committee of the Medical University of Vienna and written informed consent was obtained from parents before birth.

\footnotetext{
'Division of Neonatology, Department of Paediatrics and Adolescent Medicine, Paediatric Intensive Care and Neuropaediatrics, Medical University of Vienna, Vienna, Austria; ${ }^{2}$ Department of Surgery, Research Labs and Core Facility Flow Cytometry, Medical University of Vienna, Vienna, Austria. Correspondence: Lukas Wisgrill (lukas.wisgrill@meduniwien.ac.at)

Received 23 March 2017; accepted 9 August 2017; advance online publication 27 September 2017. doi:10.1038/pr.2017.202
} 
Isolation and Cell Culture of HUVECs

The umbilical vein was flushed twice with PBS and ECs were detached after incubation with dispase (Corning, Corning, NY) for $10 \mathrm{~min}$ at $37^{\circ} \mathrm{C}$. Detached cells were collected, the umbilical vein rinsed twice with PBS, and human umbilical vein endothelial cells (HUVECs) were pelleted by centrifugation at $300 \mathrm{~g}$ for $10 \mathrm{~min}$. The supernatant was discarded and cells were resuspended in EGM-2 medium (Lonza, Basel, Switzerland) and seeded in fibronectin (Sigma-Aldrich, St Louis, MO)-coated culture flasks. The medium was changed every 2 days and cell cultures routinely checked for growth and confluency using light microscopy. For all experiments, HUVECs between passages 2 and 6 were used.

\section{Analysis of Cytokine and Pattern Recognition Receptor Expression}

Expression of cytokine and pattern recognition receptors was analyzed on unstimulated HUVECS. Briefly, HUVECs were harvested with collagenase, washed, and stained with the following monoclonal antibodies for $20 \mathrm{~min}$ at room temperature: antiCD120a (TNFR1) fluorescein isothiocyanate (Miltenyi Biotec, Bergisch Gladbach, Germany), anti-CD120b (TNFR2) antigenpresenting cell (APC; BioLegend, San Diego, CA), anti-CD121a (IL-1R1) unconjugated (R\&D Systems, Minneapolis, MN), and antiCD284 (TLR4) phycoerythrin (PE) (eBioscience, Vienna, Austria). CD121a was secondary-labeled with an APC-conjugated Anti-Mouse IgG secondary antibody (R\&D Systems) for further $20 \mathrm{~min}$. Cells were washed twice with PBS containing $0.1 \% \mathrm{NaN}_{3} / 1 \%$ fetal calf serum and were immediately analyzed on a LSRII flow cytometer (BD Biosciences, San Jose, CA).

\section{In vitro Stimulation and Activation Marker Staining}

In all, $10^{5}$ HUVECs were seeded in fibronectin-coated 24 -well plates. After $24 \mathrm{~h}$, HUVECs were stimulated with tumor necrosis factor

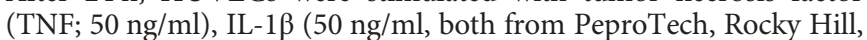
NJ), lipopolysaccharide (LPS; $10 \mu \mathrm{g} / \mathrm{ml}$, Invivogen, San Diego, CA) or were left untreated for $6 \mathrm{~h}$ in a humidified $5 \% \mathrm{CO}_{2}$ environment at $37^{\circ} \mathrm{C}$. Cells were harvested with collagenase, washed, and stained with the following monoclonal antibodies for $20 \mathrm{~min}$ at room temperature: anti-CD31 (PECAM-1) fluorescein isothiocyanate, anti-CD54 (ICAM-1) APC, anti-CD62E (E-selectin) PE, antiCD62P (P-selectin) PE, anti-CD106 (VCAM-1) PE-Cy7, and antiCD142 (tissue factor) APC (all from eBioscience). CD62P expression was analyzed at 5,10 , and $30 \mathrm{~min}$ after stimulation with histamine (50 $\mu \mathrm{m}$, Sigma-Aldrich). Cells were fixed with PFA $4 \%$, washed twice with PBS containing $0.1 \% \mathrm{NaN}_{3} / 1 \%$ fetal calf serum, and were immediately analyzed on a LSRII flow cytometer (BD Biosciences).

\section{Chemokine Production}

In all, $10^{5}$ HUVECs were seeded in fibronectin-coated 24-well plates. After $24 \mathrm{~h}$, HUVECs were stimulated with different concentrations of TNF $(2.5,10$, or $50 \mathrm{ng} / \mathrm{ml})$, IL-1 $\beta(2.5,10$, or $50 \mathrm{ng} / \mathrm{ml}$, both from PeproTech), LPS $(0.1,1$, or $10 \mu \mathrm{g} / \mathrm{ml}$, Invivogen) or were left untreated for $24 \mathrm{~h}$ in a humidified $5 \% \mathrm{CO}_{2}$ environment at $37^{\circ} \mathrm{C}$. After stimulation, the supernatant was collected and centrifuged at $300 \mathrm{~g}$ for $5 \mathrm{~min}$ at $4{ }^{\circ} \mathrm{C}$ and the cell-free supernatant was stored at $-80^{\circ} \mathrm{C}$ until further analysis. Concentrations of CCL2 (MCP-1), CCL5 (RANTES), CXCL8 (IL-8), CXCL9 (MIG), and CXCL10 (IP-10) in the cell culture supernatant were measured with Cytometric Bead Array according to the manufacturer's instructions (BD Biosciences).

\section{NFkB Phosphorylation}

In all, $10^{5}$ HUVECs were either treated with vehicle (EGM-2) or were stimulated with TNF (50 ng/ml, PeproTech) or TLR4-specific LPS $\left(10 \mu \mathrm{g} / \mathrm{ml}\right.$, Invivogen) for $15 \mathrm{~min}$ at $37^{\circ} \mathrm{C}$ in sterile pyrogen-free tubes. Cells were fixed using Phosflow Lysis/Fix Buffer (10 min at $37^{\circ} \mathrm{C}$; BD Biosciences), followed by permeabilization with ice-cold PERM III buffer (BD Biosciences) for $30 \mathrm{~min}$ on ice. Cells were washed twice and stained with anti-phospho p65 (pS529) PE (BD
Biosciences). Antibody incubation was performed for $30 \mathrm{~min}$ at room temperature. The median fluorescence intensity (MFI) was used to determine the fold change on TLR-ligand stimulation (MFIstimulated/MFI-unstimulated).

\section{Leukocyte-Endothelium Adhesion Assay}

For leukocyte adhesion experiments, assays were performed using the CytoSelect Leukocyte-Endothelium Adhesion Assay Kit (Cell Biolabs, San Diego, CA) according to the manufacturer's protocol. Briefly, $10^{5}$ HUVECs were seeded into gelatin-coated 48-well plates in EGM-2 medium until reaching confluency. Afterwards, the HUVEC monolayer was stimulated with TNF (50 ng/ml, PeproTech) or with TLR4-specific LPS $(10 \mu \mathrm{g} / \mathrm{ml}$, Invivogen $)$ for $6 \mathrm{~h}$ at $37^{\circ} \mathrm{C}$. THP-1 cells (ATCC, Manassas, VA, USA) were labeled with fluorescent LeukoTracker for $60 \mathrm{~min}$ and added to the HUVEC monolayer for $30 \mathrm{~min}$. Cells were washed twice with PBS, treated with lysing buffer, and fluorescence was measured with Varioskan Flash (Thermo Fisher, Waltham, MA).

\section{RNA Extraction and Reverse-Transcriptase Quantitative PCR} For E-selectin mRNA quantification, $10^{5}$ HUVECs were either treated with vehicle (EGM-2) or were stimulated with TNF (50 ng/ $\mathrm{ml}$, PeproTech) or TLR4-specific LPS $(10 \mu \mathrm{g} / \mathrm{ml}$, Invivogen) for $3 \mathrm{~h}$ at $37^{\circ} \mathrm{C}$. Cells were harvested and total RNA was extracted using the RNeasy Plus Mini Kit (Qiagen, Venlo, NL). DNA quantity and quality was assessed using NanoDrop8000 (Thermo Fisher). The ABI PRISM 7500HT Sequence Detection System (Applied Biosystems, Foster City, CA) was used for reverse-transcriptase quantitative PCR analysis. Primer probe sets for E-selectin FAM and 18S rRNA VIC were obtained predesigned from Applied Biosystems and were tested for primer efficacy (gene expression assays: Hs99999901_s1 18S VIC and Hs00174057_m1 SELE FAM). Multiplex amplification was carried out in a total volume of $20 \mu \mathrm{l}$ for 40 cycles of $3 \mathrm{~s}$ at $95^{\circ} \mathrm{C}$ and $30 \mathrm{~s}$ at $60^{\circ} \mathrm{C}$. Initial denaturation was performed for $3 \mathrm{~min}$ at $95^{\circ} \mathrm{C}$. Target gene expression was normalized to $18 \mathrm{~S}$ rRNA housekeeping gene expression. Normalized target gene expression was analysed by the comparative $\Delta \Delta \mathrm{CT}$ method and calculated as $x$-fold expression.

\section{Statistical Analysis}

SPSS Statistics 24.0 (IBM, Armonk, NY) was used for statistical analysis. Flow Cytometric data were analyzed using FlowJo vX (FlowJo LLC, Ashland, OR). Data were analyzed using one-way ANOVA in accordance to Tukey. A probability of $<0.05$ was considered statistically significant.

\section{RESULTS}

Adhesion and Activation Marker Expression and Function after Proinflammatory Stimuli

In the first step, we compared the effect of three different proinflammatory stimuli on the surface expression of adhesion molecules and activation markers on HUVECs in term and preterm neonates. PECAM-1 was used as an EC marker and it showed no difference in either basal expression or after stimulation between preterm and term HUVECs (data not shown). Both TNF and IL-1 $\beta$ led to a significantly higher and similar expression of ICAM-1, E-selectin, and VCAM-1 on term and preterm HUVECs. LPS induced a higher expression of E-selectin on term HUVECs compared with preterm cells. E-selectin mRNA expression was significantly lower in preterm compared with term HUVEC. In case of TNF stimulation, E-selectin mRNA showed similar expression levels (Figure 2c). Interestingly, LPS showed a slightly, but not significant, induction of ICAM-1 and VCAM-1 in term and preterm neonates. As for tissue factor 


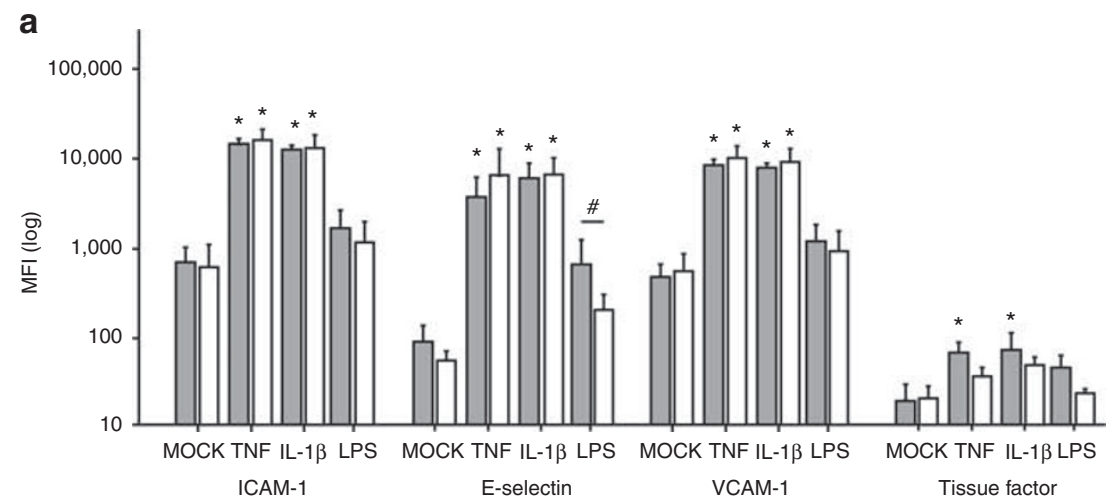

b

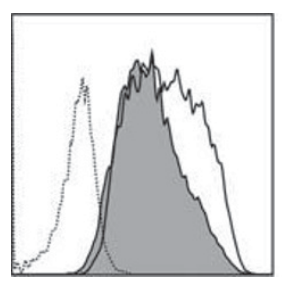

ICAM-1

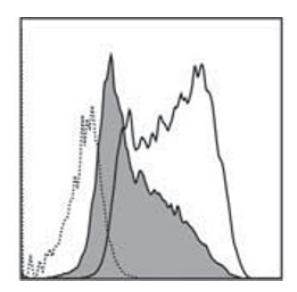

E-selectin

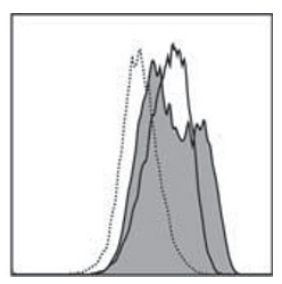

VCAM-1
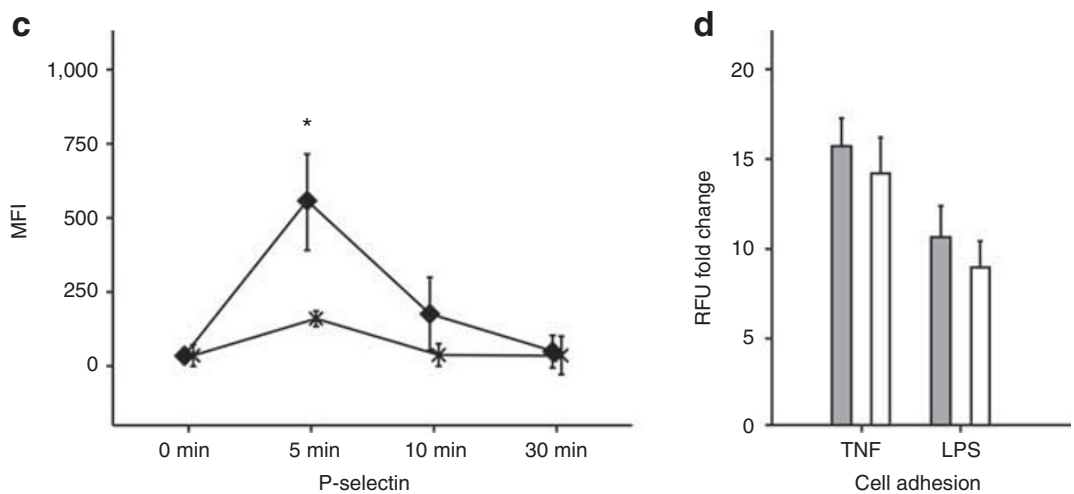

Figure 1. The effect of proinflammatory stimuli on cell surface marker expression and cell adhesion on HUVECs. HUVECs derived from preterm (white bars) and term (gray bars) newborns were stimulated with TNF, IL-1 $\beta$, and LPS, and cell adhesion molecule expression was measured using flow cytometry. Bars show mean fluorescence intensities \pm 1 SD (a). Representative histograms of cell adhesion molecule expression upon LPS stimulation in preterm (gray histogram) and term (white histogram) HUVECs compared with unstimulated control (white dotted line histogram; b). Time kinetic of P-selectin expression in preterm (X) and term ( ) HUVECs after stimulation with histamine (c). Adhesion of THP-1 cell after stimulation of HUVECs derived from preterm (white bars) and term (gray bars) newborns with TNF and LPS. Data were analyzed as fold change from fluorescence intensity of adhered THP- 1 cells on stimulated vs. unstimulated HUVECs (d). ${ }^{*} P<0.05$ vs. Mock, ${ }^{\#} P<0.05$ term vs. preterm HUVECs. HUVEC, human umbilical vein endothelial cell; IL, interleukin; LPS, lipopolysaccharide; MFI, mean fluorescence intensity; RFU, relative fluorescence unit; TNF, tumor necrosis factor.

expression, only term HUVECs displayed a significantly higher expression after TNF and IL-1 $\beta$ stimulation compared with preterm infants. LPS induced only a marginal upregulation of tissue factor on HUVECs of both age groups (Figure $\mathbf{1 a} / \mathbf{b})$. Similar to TNF, IL-1 $\beta$ and LPS did not upregulate P-selectin (data not shown). We further analyzed the rapid and dynamic expression of P-selectin after histamine exposure in a time-dependent manner from 5 , to 10 , to 30 min. HUVECs from premature infants showed a significantly decreased expression of $\mathrm{P}$-selectin compared with HUVECs from term newborns at 5 min (Figure 1c). Using a static adhesion assay, preterm HUVEC showed lower-but not significant-potential of cell adhesion compared with term HUVECs after TNF and LPS stimulation.

\section{Cytokine and TLR Receptor Expression and TLR Signaling}

In the next step, we analyzed the levels of cytokine and pathogen recognition receptors. TNFR1, TNFR2, IL-1R1, and TLR4 showed similar expression patterns in both term and preterm HUVECs (Figure 2a). Interestingly, TNF stimulation showed higher NFkB p65 phosphorylation levels than LPS in both age groups. As shown in Figure 2b, NFkB activation upon LPS stimulation increased, but not significantly, in a GA-dependent manner with highest levels in term newborns. 
a

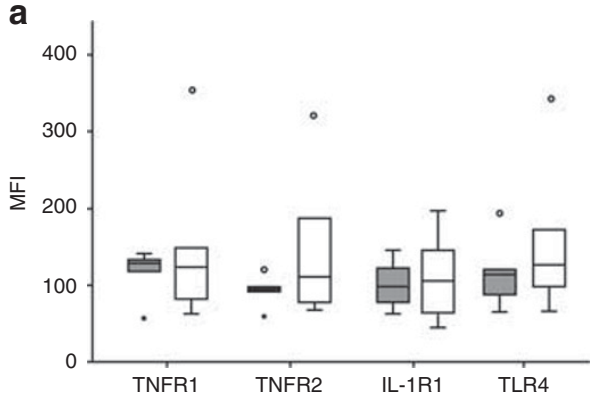

C

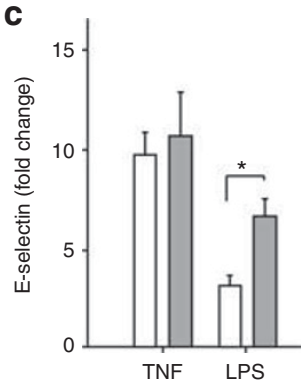

b

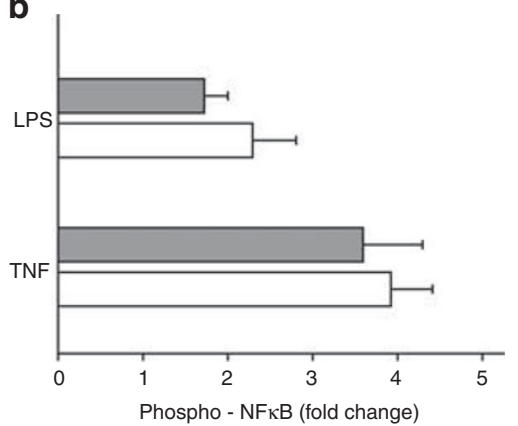

Figure 2. Cytokine receptor and pathogen pattern recognition receptor expression and NFkB signaling in HUVECs. Surface expression patterns of TNFR1, TNFR2, IL-1R1, and TLR4 of preterm (white boxplot) and term (gray boxplot) HUVECs (a). NFKB p65 phosphorylation upon LPS or TNF incubation in preterm (white bars) and term (gray bars) HUVECs (b). HUVECs from preterm (white bars) and term (gray bars) neonates were stimulated with TNF and LPS for $3 \mathrm{~h}$, and E-selectin mRNA expression was determined using RT-PCR. Values are shown as fold change in relation to unstimulated control (c). ${ }^{*} P<0.05$. HUVEC, human umbilical vein endothelial cell; IL, interleukin; LPS, lipopolysaccharide; MFI, mean fluorescence intensity; RT-PCR, reverse-transcriptase PCR; TLR, Toll-like receptor; TNF, tumor necrosis factor.

Moreover, phosphorylation of NFKB after LPS stimulation appeared to be deficient in preterm neonates. Stimulation with TNF resulted in similar phosphorylation of NFKB in both age groups (Figure $\mathbf{2 b}$ ).

\section{Chemokine Production after Proinflammatory Stimulation}

To determine the effect of proinflammatory stimuli on the chemokine production in human ECs, HUVECs were incubated with various concentrations of TNF, IL-1 $\beta$, and LPS for $24 \mathrm{~h}$. Both CCL2 and CXCL8 were significantly induced upon proinflammatory stimulation and in comparable concentrations in preterm and term HUVECs (Figure $3 \mathrm{a} / \mathrm{c}$ ). Low levels of CCL5 were observed in both age groups upon higher dosage of TNF. Neither challenge with IL-1 $\beta$ nor with LPS resulted in the synthesis of CCL5 (Figure 3b). In contrast, CXCL10 was upregulated in HUVECs upon stimulation with TNF with higher levels in term infants compared with preterm infants. IL-1 $\beta$ and LPS had no influence on the secretion of CXCL10 in preterm and term HUVECs (Figure 3d). In both groups, CXCL9 production was not induced after stimulation with the mentioned stimuli (data not shown).

\section{DISCUSSION}

Results of our study demonstrate partly reduced expression levels and function of cell adhesion molecules, as well as reduced NFKB signaling and chemokine/cytokine production upon stimulation in ECs of extremely premature infants compared with term infants. This diminished endothelial proinflammatory immune response might contribute to the impaired infection control of preterm newborns rendering them prone to severe infections.

The recruitment, activation, and extravasation of leukocytes to sites of infection are a key mechanism in the immune response to defend invading microorganism. ECs have an important role in the inflammatory immune response during systemic infections by producing proinflammatory cytokines as well as chemokines facilitating extravasation of immune cells by expression of adhesion molecules $(9,10)$. Extravasation is mediated by the surface expression of cellular adhesion molecules on ECs. Adhesion molecules such as selectins (E-Selectin), integrins, and immunoglobulin-like adhesion molecule (ICAM-1, VCAM-1) have a key role in leukocyte/ endothelial activation via out-in-signaling (11), furthermore, facilitating leukocyte rolling, adherence, and transmigration of different immune cells such as monocytes, neutrophils, or $\mathrm{T}$ cells (12). Notably, all studies analyzing the immune response of ECs under proinflammatory conditions investigated HUVECs of term neonates so far. Although those studies have deepened our knowledge of the physiology and pathophysiology of HUVECs under inflammatory conditions, our understanding of EC function in extremely premature infants remains unknown. Therefore, in the present study we investigated the immune response of 
HUVECs from extremely preterm newborns and compared their surface receptor expression, adhesive functions, cell signaling, and chemokine production with those from term newborns.
Cell adhesion molecules constitutively being expressed on the cell surface of HUVECs mediate binding and transmigration of leukocytes through the endothelial wall. The qualitative and quantitative expression of selectin ligands/integrins on target
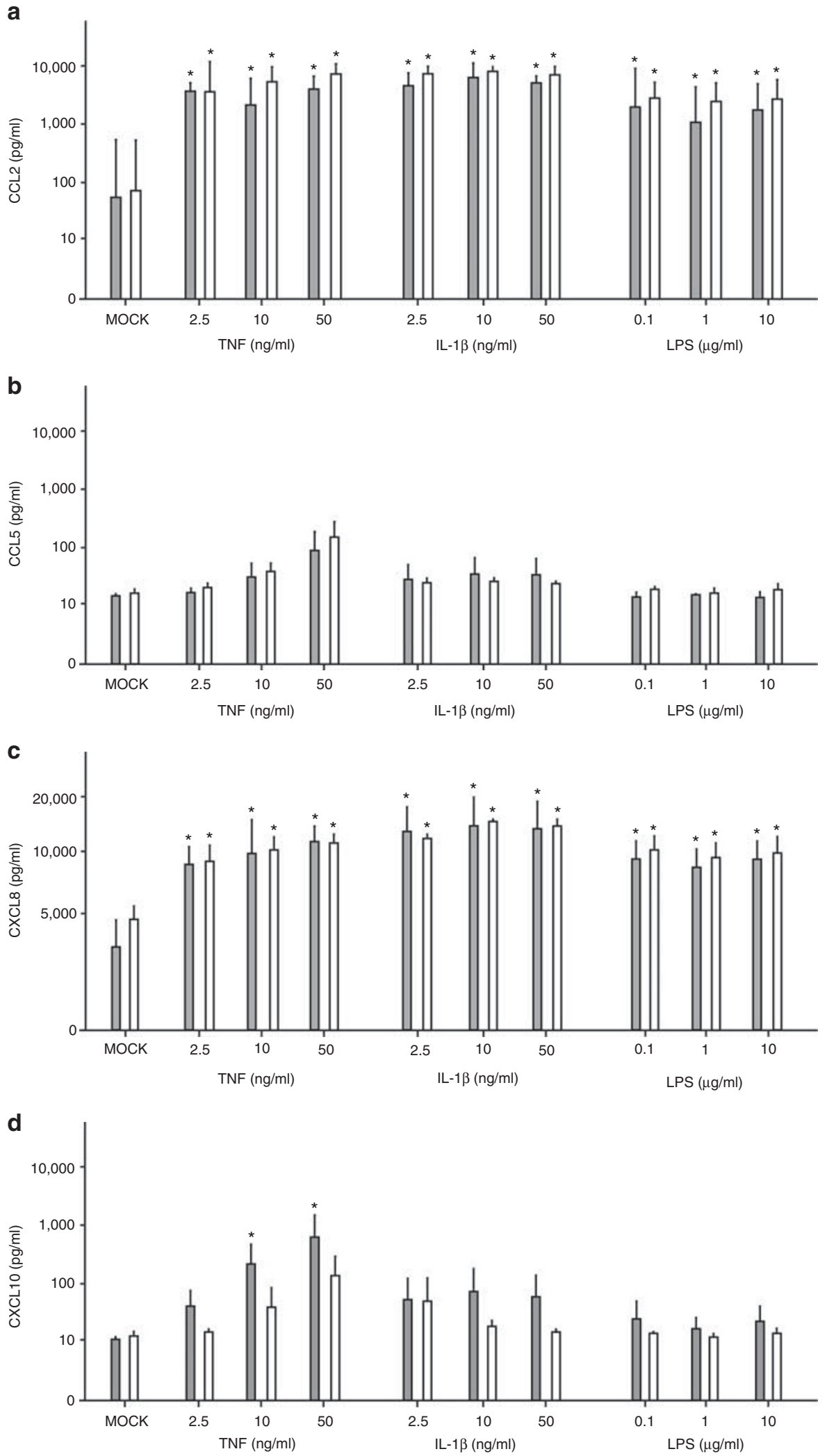
cells has a major role for successful binding and migration $(13,14)$. In premature neonates, both selectin expression on ECs as well as selectin-ligand/integrin expression on leukocytes seem to be responsible for the reduced recruitment of leukocytes toward the site of inflammation $(15,16)$. In our study, the expression of the cell adhesion molecules ICAM-1, E-selectin, and VCAM-1 upon LPS stimulation differed in term and extremely preterm infants, showing a significantly lower expression of E-selectin-on protein and transcriptional levels -and a marginally lower expression of ICAM- 1 and VCAM- 1 in preterm HUVECs. Interestingly, we found comparable expression of selectins in both age groups after stimulation with TNF and IL-1 $\beta$. In addition, P-selectin, which is stored in Weibel-Pallade bodies and rapidly released after histamine stimulation, was nearly absent in extremely premature HUVECs. These findings correlate with electron microscopic analyses showing a decreased number of Weibel-Pallade bodies in premature ECs $(17)$. Likewise, we and others $(15,18,19)$ found a GA-dependent correlation of selectin expression of HUVECs after proinflammatory stimulation. This deficiency of selectin expression in premature ECs seems to be further aggravated by significant differences in selectin-ligand and integrin expression on neonatal leukocytes. Neonatal neutrophils, especially those of infants born before 30 weeks of GA, express lower basal levels of L-selectin, PSGL-1, and CD11b/CD18 as well as defective upregulation of $\mathrm{CD} 11 \mathrm{~b} / \mathrm{CD} 18$ after proinflammatory stimulation. Furthermore, Nussbaum et al. (15) used flow chamber experiments to analyze the adhesive properties of premature ECs and neutrophils and showed a decreased capability of adult neutrophil rolling on premature ECs as well as of premature neutrophils on adult ECs. These findings demonstrate the importance of both compartments, underlining the deficiency in leukocyte recruitment in premature infants. Unfortunately, we have no possibility of conducting flow chamber experiments in our laboratory. Therefore, we used a static cell adhesion assay to determine the influence of proinflammatory stimuli on the adhesive capacity of premature HUVECs. Although we found lower adhesive capacity of premature HUVECs compared with those from term newborns, the difference was not statistically significant. This might be due to the static approach we used in our experiments, underlining the importance of flow chamber assays to determine, under more physiological conditions, the influence of adhesion molecules on the cell surface.

As leukocyte recruitment also involves chemotactic stimulation, we analyzed the secretion of relevant chemokines in extremely premature and term HUVECs. The immaturity of premature ECs seems to partly influence chemokine production. Surprisingly, CCL2 and CXCL8 were significantly upregulated in extremely premature ECs and displayed comparable levels to term ECs after stimulation with TNF,
IL-1 $\beta$ and LPS. As CXCL8 is a key chemoattractant for neutrophils, the deficient rolling in flow chamber experiments might be due to lower response towards that cytokine (15). Studies investigating chemokine receptor expression and associated activation of preterm leukocytes are missing. In the context of immaturity, it has been shown that the LPSmediated activation of neonatal monocytes as well as neutrophils is diminished in preterm and term cord blood, affecting several immunological functions of those cells, which might explain the insufficient response of neonatal monocytes and neutrophils towards attracting chemokines $(15,20,21)$. In our study, secretion of CXCL10 was significantly upregulated with increasing concentrations of TNF only in term infants. None of the used stimuli induced significant production of CCL5 in HUVECs. However, others have shown that the combination of TNF and interferon- $\gamma$ was able to induce a strong upregulation of CCL5 production (22). The fetal immune response is mainly driven toward Th2-skewing cytokines resulting from a decreased interferon$\gamma$ production by NK cells and, furthermore, from diminished production of IL-12 and IL-18 from APCs (23-25). This can be regarded as one of the mechanisms of reduced recruitment of leukocytes such as monocytes in preterm infants.

One reason for the differences between the effects of the stimulants used in our experiments is that the three molecules signal through different receptors with unique expression patterns and signal transduction pathways (26). All three receptors were expressed on HUVECs of both preterm and term neonates, and no significant differences in expression between the two GA groups could be found. To deepen our analysis, we performed signaling experiments after LPS and TNF stimulation by analyzing the NFKB phosphorylation. Surprisingly, we found reduced levels of NFKB after stimulation in preterm compared with term HUVECs, although those differences were not found to be significant. The question arises whether this slight difference might influence the different phenotypes of preterm ECs, or whether other factors, such as epigenetic regulations, might influence the different response in premature infants.

In conclusion, our study sheds light on the proinflammatory response of ECs isolated from extremely premature infants and shows differences in cell adhesion molecule expression levels as well as NFKB signaling and chemokine/ cytokine production of ECs from extremely premature compared with term infants. Getting a deeper look in the physiology and pathophysiology of the immune system of extremely premature infants might reveal novel mechanisms in EC inflammation and, furthermore, open the possibility to investigate the role of ECs as immune cells in different in vitro settings such as brain injury or necrotizing enterocolitis.

Figure 3. Chemokine secretion after proinflammatory stimulation of HUVECs. Dose-dependent secretion of CCL2 (a), CCL5 (b), CXCL8 (c), and CXCL10 (d) in HUVECs derived from preterm (white bars) and term (gray bars) newborns after stimulation with TNF, IL-1 $\beta$, and LPS. Bars show the mean cytokine concentrations $\pm 1 \mathrm{SD}$. ${ }^{*} P<0.05$ vs. medium-only control (Mock). HUVEC, human umbilical vein endothelial cell; IL, interleukin; LPS, lipopolysaccharide; TNF, tumor necrosis factor. 


\section{Articles | Wisgrill et al.}

Further and deepened research is necessary to understand the precise mechanisms behind this immaturity, thereby potentially unraveling novel mechanisms to positively influence the immune response of extremely premature neonates.

\section{ACKNOWLEDGMENTS}

We want to thank Günther Hofbauer, Core Unit for Flow Cytometry, Medical University of Vienna, for technical assistance.

\section{STATEMENT OF FINANCIAL SUPPORT}

This work was supported by in-house funding from our institution.

Disclosure: The authors declare no conflict of interest.

\section{REFERENCES}

1. Stoll BJ, Hansen NI, Sanchez PJ, et al. Early onset neonatal sepsis: the burden of group B Streptococcal and E. coli disease continues. Pediatrics 2011;127:817-26.

2. Simonsen KA, Anderson-Berry AL, Delair SF, Davies HD. Early-onset neonatal sepsis. Clin Microbiol Rev 2014;27:21-47.

3. Cortese F, Scicchitano P, Gesualdo M, et al. Early and late infections in newborns: where do we stand? A review. Pediatr Neonatol 2015;57: 265-73.

4. Gupta SK, Lysko PG, Pillarisetti K, Ohlstein E, Stadel JM. Chemokine receptors in human endothelial cells. Functional expression of CXCR4 and its transcriptional regulation by inflammatory cytokines. J Biol Chem 1998;273:4282-7.

5. Mitchell JA, Ryffel B, Quesniaux VF, Cartwright N, Paul-Clark M. Role of pattern-recognition receptors in cardiovascular health and disease. Biochem Soc Trans 2007;35:1449-52.

6. Murdoch C, Monk PN, Finn A.. Cxc chemokine receptor expression on human endothelial cells. Cytokine 1999;11:704-12.

7. Opitz B, Eitel J, Meixenberger K, Suttorp N. Role of Toll-like receptors, NOD-like receptors and RIG-I-like receptors in endothelial cells and systemic infections. Thromb Haemost 2009;102:1103-9.

8. Michiels C. Endothelial cell functions. J Cell Physiol 2003;196:430-43.

9. Kofler S, Nickel T, Weis M. Role of cytokines in cardiovascular diseases: a focus on endothelial responses to inflammation. Clin Sci 2005;108: 205-13.

10. Cines DB, Pollak ES, Buck CA, et al. Endothelial cells in physiology and in the pathophysiology of vascular disorders. Blood 1998;91:3527-61.
11. Lorenzon P, Vecile E, Nardon E, et al. Endothelial cell E- and P-selectin and vascular cell adhesion molecule-1 function as signaling receptors. J Cell Biol 1998;142:1381-91.

12. Golias C, Batistatou A, Bablekos G, et al. Physiology and pathophysiology of selectins, integrins, and IgSF cell adhesion molecules focusing on inflammation. A paradigm model on infectious endocarditis. Cell Commun Adhes 2011;18:19-32.

13. McEver RP. Selectins: initiators of leucocyte adhesion and signalling at the vascular wall. Cardiovasc Res 2015;107:331-9.

14. Muller WA. Leukocyte-endothelial-cell interactions in leukocyte transmigration and the inflammatory response. Trends Immunol 2003;24:327-4.

15. Nussbaum C, Gloning A, Pruenster M, et al. Neutrophil and endothelial adhesive function during human fetal ontogeny. J Leukoc Biol 2013;93: $175-84$.

16. Nussbaum C, Sperandio M. Innate immune cell recruitment in the fetus and neonate. J Reprod Immunol 2011;90:74-81.

17. Lorant DE, Li W, Tabatabaei N, Garver MK, Albertine KH. P-selectin expression by endothelial cells is decreased in neonatal rats and human premature infants. Blood 1999;94:600-9.

18. Dogan A, MacDonald TT, Spencer J. Expression of cell adhesion molecules in the fetal gut. Adv Exp Med Biol 1995;371A:141-3.

19. Mariscalco MM, Tcharmtchi MH, Smith CW. P-Selectin support of neonatal neutrophil adherence under flow: contribution of L-selectin, LFA-1, and ligand (s) for P-selectin. Blood 1998;91:4776-85.

20. Schuller S, Wisgrill L, Sadeghi K, et al. The TLR-specific adjuvants R-848 and $\mathrm{CpG}-\mathrm{B}$ endorse the immunological reaction of neonatal antigen presenting cells. Pediatr Res 2016;80:311-8.

21. Wisgrill L, Groschopf A, Herndl E, et al. Reduced TNF-alpha response in preterm neonates is associated with impaired nonclassic monocyte function. J Leukoc Biol 2016;100:607-12.

22. Marfaing-Koka A, Devergne O, Gorgone G, et al. Regulation of the production of the RANTES chemokine by endothelial cells. Synergistic induction by IFN-gamma plus TNF-alpha and inhibition by IL-4 and IL-13. J Immunol 1995;154:1870-8.

23. Garcia AM, Fadel SA, Cao S, Sarzotti M. T cell immunity in neonates. Immunol Res 2000;22:177-90.

24. Lavoie PM, Huang Q, Jolette E, et al. Profound lack of interleukin (IL)-12/ IL-23p40 in neonates born early in gestation is associated with an increased risk of sepsis. J Infect Dis 2010;202:1754-63.

25. Sharma AA, Jen R, Brant R, et al. Hierarchical maturation of innate immune defences in very preterm neonates. Neonatology 2014;106:1-9.

26. Mako V, Czucz J, Weiszhar Z, et al. Proinflammatory activation pattern of human umbilical vein endothelial cells induced by IL-1beta, TNFalpha, and LPS. Cytometry A 2010;77:962-70. 